\begin{tabular}{|c|l|}
\hline Title & Macroscopic and microscopic hydrodynamic mixing of stratified suspensions \\
\hline Author(s) & Y amamoto, Y asufumi; Y amada, Koki; Tanaka, Y ohsuke; Harada, Shusaku \\
\hline Citation & $\begin{array}{l}\text { Physical Review E, 104(2), 025111 } \\
\text { https://doi.org/10.1103/PhysRevE.104.025111 }\end{array}$ \\
\hline Issue Date & 2021-08-27 \\
\hline Doc URL & http://hdl.handle.net/2115/82805 \\
\hline Rights & Copyright (2021) by The A merican Physical Society. \\
\hline Type & article \\
\hline File Information & PhysRevE.104.025111.pdf \\
\hline
\end{tabular}

Instructions for use 


\title{
Macroscopic and microscopic hydrodynamic mixing of stratified suspensions
}

\author{
Yasufumi Yamamoto $\odot^{*}$ and Koki Yamada \\ Department of Mechanical Engineering, Kansai University, 3-35 Yamate-cho 3-chome, Suita, Osaka 564-8680, Japan \\ Yohsuke Tanaka \\ Faculty of Mechanical Engineering, Kyoto Institute of Technology, Matsugasaki, Sakyo-ku, Kyoto 606-8585, Japan
}

Shusaku Harada $\odot$

Division of Sustainable Resources Engineering, Faculty of Engineering, Hokkaido University, N13-W8 Sapporo, Hokkaido 060-8628, Japan

(Received 24 June 2021; accepted 17 August 2021; published 27 August 2021)

\begin{abstract}
We conducted numerical experiments to investigate the mixing of stratified suspensions containing different types of particles. We used a point-force two-way coupling method. We studied the mixing behavior of stratified suspensions and we discovered two types of mixing: microscopic (individual-particle-level) and macroscopic (vessel-scale) collective mixing. In addition, we examined the vertical mixing speed of the stratified suspension. We used a simple theoretical model to analyze the fingering settling velocity. Then we introduced a nondimensional number representing the difference in collectivities of the upper and lower suspensions while accounting for particle terminal velocities. We discovered that the proposed nondimensional parameter has a negative sign that distinguishes the mixing form of only microscopic individual-particle-level mixing and a positive value that predicts the speed of macroscopic collective mixing of stratified suspensions.
\end{abstract}

DOI: 10.1103/PhysRevE.104.025111

\section{INTRODUCTION}

The mixing of particulate matters in liquid is a common phenomenon in chemical, civil, environmental, and mechanical engineering processes. While there are processes that promote the mixing of unevenly dispersed particles, some processes inhibit mixing, such as the separation or stratification of different types of particles. It is critical to quantitatively predict and control the mixing behaviors for a given particle and fluid condition in both processes.

In general, the mixing of nanoparticles or macromolecules is explained in a thermodynamic framework as an increase in entropy due to the lack of gravity's influence and the dominance of thermal motion. According to Fick's law, mixing these particles occurs via a mass flux proportional to the concentration gradient. The mixing of micro-sized particles in liquid, on the other hand, has an entirely different mechanism from the Fickian mixing. The hydrodynamic force intricately affects the relative motion of particles in such a system and, as a result, mixing occurs on various spatial scales.

Consider two types of micro-sized particle suspensions that are stratified in an ideal state. If the upper and lower particles settle independently, each particle settles at the hindered settling velocity determined by the mass density, particle size, and concentration [1]. As a result of the difference in settling velocities on a spatial scale at the particle level, the upper and lower particles mix. The particle mixing caused by the difference in settling velocity can be observed in stratified

*yamayasu@kansai-u.ac.jp suspensions where the concentration gradient is positive in the direction of gravity [2].

In contrast, suspensions with an interface where the concentration gradient is negative with respect to the direction of gravity cause large-scale convective motion due to gravitational instability at the concentration interface. It causes a wide variety of exciting suspension motions, such as the convection formation in vessels known as the Boycott effect [3], fingerlike settling of suspensions [4,5], and the splitting of suspension droplets such as with fireworks [6-8].

In the motion mentioned above, the suspended particles sometimes behave as a continuous fluid. The fluid flow induced by the individual particles causes this collective motion, which appears to shield the interface that borders the particles, forming an immiscible interface [9]. The dimensionless parameter collectivity, which describes particle resolution of the concentration interface, determines the collective motion of suspension [5]. Suspended particles behave as a continuous fluid with high collectivity, whereas they behave as individual particles with low collectivity [5].

Under high collectivity conditions, gravitational instability would cause the mixing of stratified suspensions with a negative concentration gradient on a large spatial scale. It is similar to the miscible and immiscible fluids generated by gravitational instability, and it has long been studied as Rayleigh-Taylor mixing (RT mixing). In these studies, it was demonstrated that the growth speed of fingerlike interface instability can be expressed similarly to the bubble rising velocity [10]. The growth rate of RT mixing in the infinite region exhibits a time dependency due to lateral finger growth [11-16]. Theoretical, experimental, and numerical studies of 
single-mode RT mixing in a container with limited dimensions have revealed that the mixing speed becomes constant as a function of fluid density, viscosity, and interfacial tension [17-21]. Like RT mixing of ordinary fluids, mixing of stratified suspensions with high collectivity is expected to occur on a large scale.

As previously stated, the hydrodynamic mixing of microsized particles is considered to be far from the Fickian mixing, which is determined by the difference in settling velocity and gravitational instability depending on the suspension conditions. It is critical in engineering to understand the conditions under which the microscopic and macroscopic mixing described above occurs between the suspended particles, as well as what determines the mixing speed.

Mori et al. [22] used experiments and numerical simulations to investigate the mixing behavior of stratified suspensions with different volume fractions (i.e.,different collectivities) composed of identical particles by gravitational instability. It was discovered that the settling velocity of the upper suspension can be determined by the square difference between the collectivities of the upper and lower suspensions, and that the hydrodynamic mixing of micro-sized particles exhibits non-Fickian characteristics.

In this study, we conducted numerical experiments on mixing stratified suspensions containing various types of particles. Our previous study [22] has been extended to investigate the hydrodynamic mixing of micro-sized particles under various conditions such as collectivity difference, apparent mass density difference, and single-particle terminal velocity difference of the upper and lower suspensions. As expected from previous studies [5,22], the high collectivity of the upper suspension enhances large-scale mixing via gravitational instability. In contrast, the high terminal velocity of the upper suspension enhances small-scale mixing via invasion to lower suspension across the concentration interface. We investigate such multiscale mixing of stratified suspension at the concentration interface using numerical simulations with various suspension conditions and discuss the mixing speed quantitatively using a nondimensional number.

\section{SYSTEM AND SIMULATION}

The schematic representation of the numerical experiment system is shown in Fig. 1. The test cell is a rectangular vessel

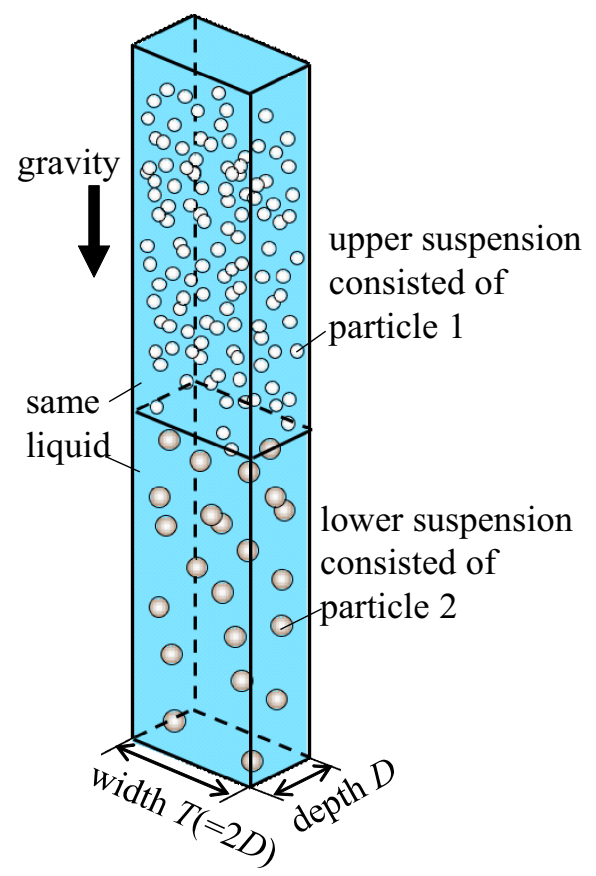

FIG. 1. Configuration of stratified suspensions in rectangular vessel.

with $D=3 \mathrm{~mm}$ and $T=2 D$. The height $L$ is conditionally adjusted. Stationary suspensions are initially installed on the upper and lower sides. The liquid's properties are set similar to those of silicone oil (mass density $\rho_{l}=972 \mathrm{~kg} / \mathrm{m}^{3}$ and dynamic viscosity $\mu_{l}=100 \mathrm{mPa}$ ). The upper suspension's initial height ranges from $2.3 D$ to $4.6 D$, and the lower suspension's initial height ranges from $3.45 D$ to $4.6 D$. Except for the particle condition, these configurations are identical to Mori et al. [22]. The properties of particles vary, as shown in Table I. The Stokes terminal velocity of a single particle $U_{\mathrm{St}}$ can be calculated as follows:

$$
U_{\mathrm{St}}=\frac{\left(\rho_{\mathrm{p}}-\rho_{l}\right)}{18 \mu_{l}} d_{\mathrm{p}}^{2} g,
$$

where $\rho_{\mathrm{p}}$ denotes the particle mass density, $d_{\mathrm{p}}$ denotes particle diameter, and $g$ is the gravitational acceleration. Based on the nondimensional number derived by Harada et al. [5,23], the collectivity $C$ has been defined as $C \equiv\left(D / d_{\mathrm{p}}\right) \phi^{1 / 3}$ by

TABLE I. Numerical condition of upper and lower suspensions for five characteristic cases.

\begin{tabular}{|c|c|c|c|c|c|c|c|c|}
\hline Case & Position & $\begin{array}{l}\text { Diameter } \\
d_{\mathrm{p}}(\mu \mathrm{m})\end{array}$ & $\begin{array}{c}\text { Density } \\
\rho_{\mathrm{p}}\left(\mathrm{kg} / \mathrm{m}^{3}\right)\end{array}$ & $\begin{array}{c}\text { Volume fraction } \\
\phi\end{array}$ & $\begin{array}{l}\text { Terminal velocity } \\
U_{\mathrm{St}}(\mu \mathrm{m} / \mathrm{s})\end{array}$ & $\begin{array}{l}\text { Apparent density } \\
\rho_{\mathrm{s}}\left(\mathrm{kg} / \mathrm{m}^{3}\right)\end{array}$ & $\begin{array}{c}\text { Collectivity } \\
\qquad C\end{array}$ & $\begin{array}{c}\text { Parameter } \\
Y_{\mathrm{m}}\end{array}$ \\
\hline \multirow[t]{2}{*}{ No. 1} & upper & 45 & 2500 & 0.01 & 16.82 & 990.3 & 14.36 & 43.8 \\
\hline & lower & 90 & 1000 & 0.009 & 1.10 & 975.2 & 6.93 & \\
\hline \multirow[t]{2}{*}{ No. 2} & upper & 67.5 & 2500 & 0.038 & 37.86 & 1033 & 17.94 & 72.3 \\
\hline & lower & 22.5 & 2500 & 0.0014 & 4.21 & 977.1 & 14.92 & \\
\hline \multirow[t]{2}{*}{ No. 3} & upper & 180 & 2500 & 0.04 & 269.2 & 1036 & 5.70 & 8.33 \\
\hline & lower & 45 & 2500 & 0.01 & 16.82 & 990.3 & 14.36 & \\
\hline \multirow[t]{2}{*}{ No. 4} & upper & 90 & 2500 & 0.001 & 37.30 & 976.5 & 3.33 & -10.0 \\
\hline & lower & 45 & 2500 & 0.01 & 16.82 & 990.3 & 14.36 & \\
\hline \multirow[t]{2}{*}{ No. 5} & upper & 90 & 2500 & 0.015 & 67.30 & 997.9 & 8.22 & 5.56 \\
\hline & lower & 45 & 2500 & 0.01 & 16.82 & 990.3 & 14.36 & \\
\hline
\end{tabular}




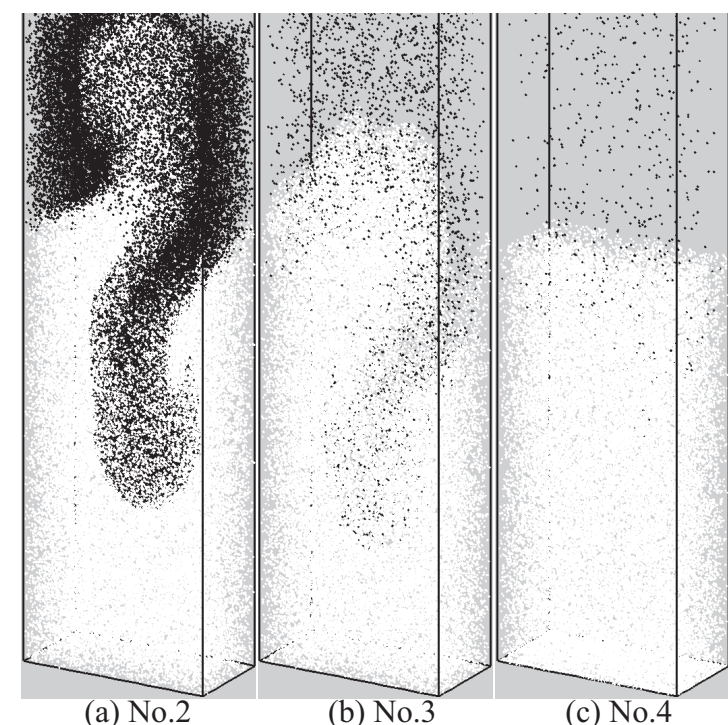

(a) No.2

(b) No.3

(c) No.4

FIG. 2. External view of settling behavior of stratified suspensions for three cases shown in Table I (black dot: upper particle; white dot: lower particle; the dot size does not reflect the size of the particle).

considering particle mean distance as described in Mori et al. [22]. When $C$ is greater than 14 (fluidlike condition), the suspended particles behave perfectly as a continuum. When $C$ values are less than 2 , they settle down almost with the terminal velocity of an isolated particle. For $C$ values between 2 and 14, the suspended particles' individual and collective behaviors coexist (intermediate condition). Unlike Mori et al., the single-particle terminal velocity of the upper suspension in this study differs from that of the lower suspension.

As in previous studies [7,22,24], we employed Lagrangian tracking of individual particles with two-way coupling using a point-force model. We could ignore particle inertia by assuming that the particle response time was much shorter than the liquid flow timescale, and thus the Stokes number was much smaller than unity. In this case, we considered particle motion resulting only from Stokes drag and gravity, without inertia, as

$$
\mathbf{0}=-3 \pi \mu_{l} d_{\mathrm{p}}\left[\boldsymbol{v}-\boldsymbol{u}\left(\boldsymbol{x}_{\mathrm{p}}\right)\right]+\frac{\pi}{6} d_{\mathrm{p}}^{3}\left(\rho_{\mathrm{p}}-\rho_{l}\right) \boldsymbol{g},
$$

where $\boldsymbol{v}$ and $\boldsymbol{u}$ are the particle velocity and liquid velocity at particle position $\boldsymbol{x}_{\mathrm{p}}$, respectively. The particle velocity was instantaneously solved using the above equation with the interpolated liquid velocity at the particle position, and the particle position was subsequently updated by integration. We used the second-order Adams-Bashforth method for integrating the particle position.

The liquid was assumed to be incompressible and its motion described by the continuity equation

$$
\nabla \cdot \boldsymbol{u}=0
$$

and the two-way-coupled Navier-Stokes equation,

$$
\frac{\partial \boldsymbol{u}}{\partial t}+\nabla \cdot \boldsymbol{u} \boldsymbol{u}=-\frac{1}{\rho_{l}} \nabla p+\frac{\mu_{l}}{\rho_{l}} \nabla^{2} \boldsymbol{u}+\frac{1}{\rho_{l}} \boldsymbol{f},
$$

where $f$ is the feedback force per unit volume as given by

$$
\boldsymbol{f}(\boldsymbol{x})=-\frac{3 \pi \mu_{l} d_{\mathrm{p}}}{\Delta^{3}} \sum_{\boldsymbol{x}_{\mathrm{p}}}\left[\boldsymbol{u}\left(\boldsymbol{x}_{\mathrm{p}}\right)-\boldsymbol{v}\right] w\left(\boldsymbol{x}-\boldsymbol{x}_{\mathrm{p}}\right),
$$

where $\boldsymbol{x}$ denotes grid position and $\Delta$ denotes grid spacing. The function $w$ is a nondimensional weighting function and it gives the trilinear distribution to the eight neighboring points as shown in [24]. The liquid flow field was obtained by solving Eqs. (3) and (4) with Eq. (5). Equations (3) and (4) were spatially discretized using a staggered-grid system and the second-order central finite-difference method. The advection term was advanced in time using the second-order AdamsBashforth method, while the viscous term was advanced using the second-order Crank-Nicolson scheme. The simplified marker and cell algorithm were used to link pressure to Eq. (3), and the Poisson equation for pressure correction was solved using the biconjugate gradient stabilized method.

The number of grid points for $D, T$, and $L$ was set to 8,16 , and 64-258, respectively. The time step was set to a range of $1.5-10 \times 10^{-5} \mathrm{~s}$. The number of particles treated under the maximum condition was 114591 and the computational time for that case was about 26 days. The particle and liquid velocities were set to zero as initial conditions, and the particle positions were determined using random numbers. The suitable implementation of the method was discussed in Yamamoto et al. [24] and was validated by quantitatively comparing the results with the experimentally obtained values as shown in Mori et al. [22]. The grid resolution dependency was checked and is shown in the Appendix.

\section{RESULT AND DISCUSSION}

\section{A. Settling behavior of stratified suspension}

Figure 2 shows the settling behavior of stratified suspensions for the No. 2(a), No. 3(b), and No. 4(c) cases in Table I: (a) high collective suspension of large particles on

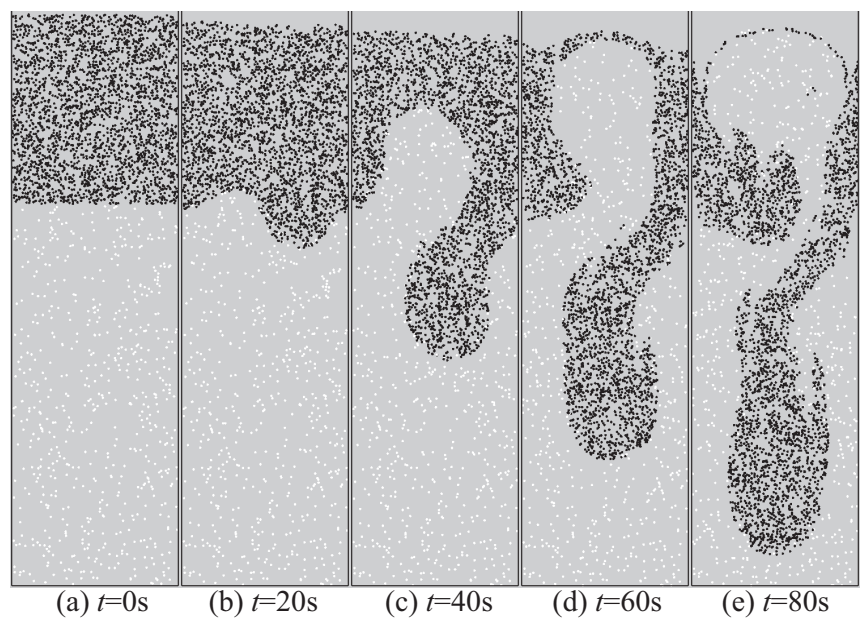

FIG. 3. Mixing behavior of high collective suspension of small heavy particles on low collective suspension of large light particles, case No. 1 (in center plane region with depth of $0.1 D$; black dot: upper particle; white dot: lower particle; the dot size does not reflect the size of the particle). 


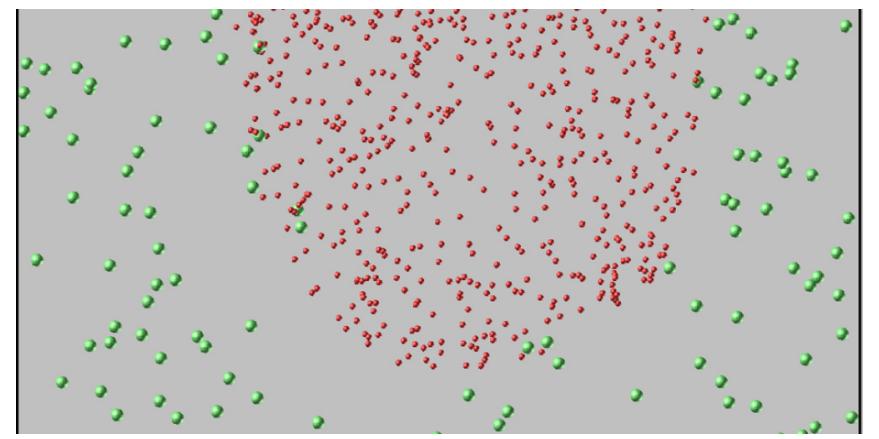

FIG. 4. Partial magnified image around the fingertip of Fig. 3(d) (scale rate of sphere image corresponds to particle size).

high collective suspension of small particle, (b) low collective suspension of large particles on high collective suspension of small particle, and (c) low collective suspension of large particles with relatively low apparent mass density on high collective suspension of small particle with relatively high apparent mass density. In case No. 2, we can see the typical fingering settling of the upper suspension due to the gravitational instability. Also, in case No. 3, fingering settling appears to occur; however, due to the low collectivity of the upper suspension, the upper particles individually invade the lower suspensions. Because it is unclear from the external view only, we will investigate the central plane region in detail later. Because the terminal velocity of the upper particle is greater than that of the lower particle, fingering settling does not occur in case No. 4, which is the gravitationally stable condition, and particle scale mixing can occur.

\section{B. Microscopic and macroscopic mixing}

This section gives a detailed discussion of the results for each case. Figure 3 shows the mixing behavior of a high collective $(C=14.36)$ suspension of small heavy particles on a low collective $(C=6.93)$ suspension of large light particles, which is case No. 1 in Table I, and Fig. 4 depicts

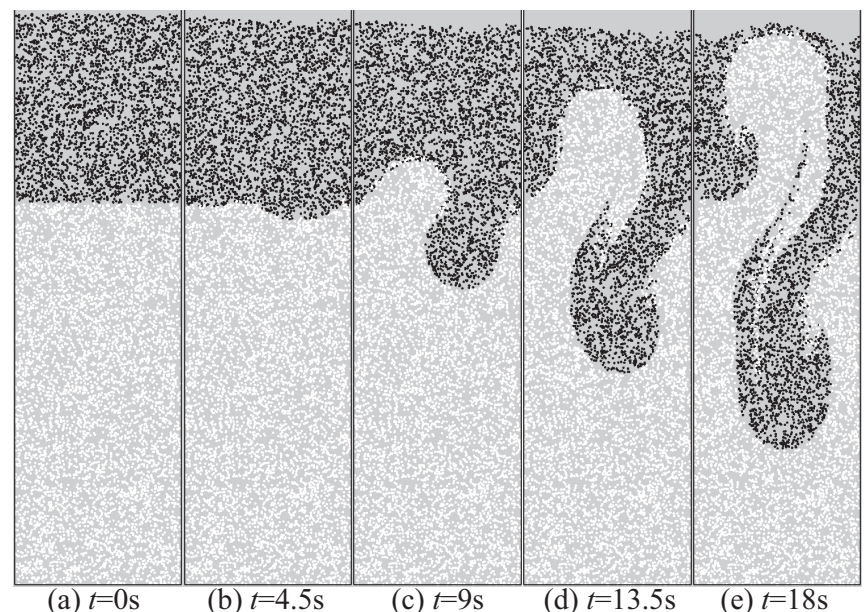

FIG. 5. Mixing behavior of high collective suspension of large particles on high collective suspension of small particles, case No. 2 (see the caption of Fig. 3).

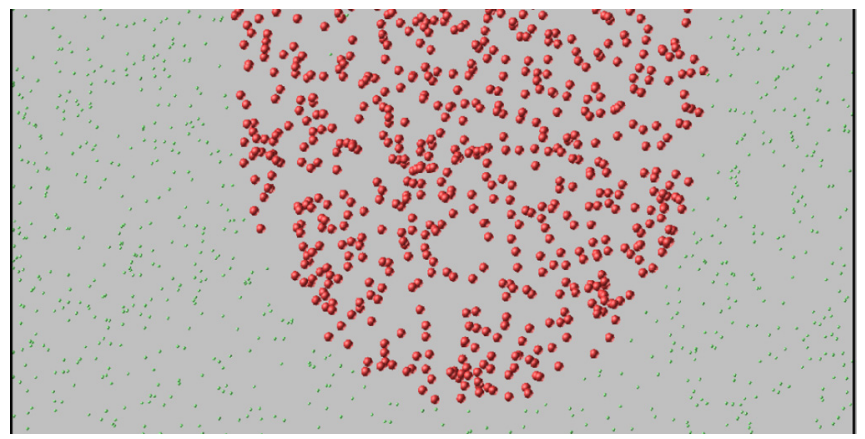

FIG. 6. Partial magnified image around the fingertip of Fig. 5(e) (see the caption of Fig. 4).

a partial magnified image around the fingertip of Fig. 3(d). Because of the gravitational instability, we can see the typical mushroom-type fingering settling of the upper suspension. In addition, due to volume exchange, the lower suspension rises. The relatively clear interface between the upper and lower suspensions can be seen by looking at the tip of the upper suspension's finger. The terminal velocity of the upper particle is greater than that of the lower particle in this case, but microscopic (individual-particle-level) mixing is rare. This is due to the high collectivity $C$ of the upper suspension. The lower particle cannot enter the upper suspension, which is made up of collective particles associated with Stokes flow fields.

Figure 5 shows the mixing behavior of a high collective $(C=17.94)$ suspension of large particles on a high collective $(C=14.92)$ suspension of small particles in case No. 2 , and Fig. 6 is a partial magnified version of Fig. 5(e). We can see the typical mushroom-type fingering settling of the upper suspension and moving up of the lower suspension due to volume exchange in this case as well. The very clear interface between

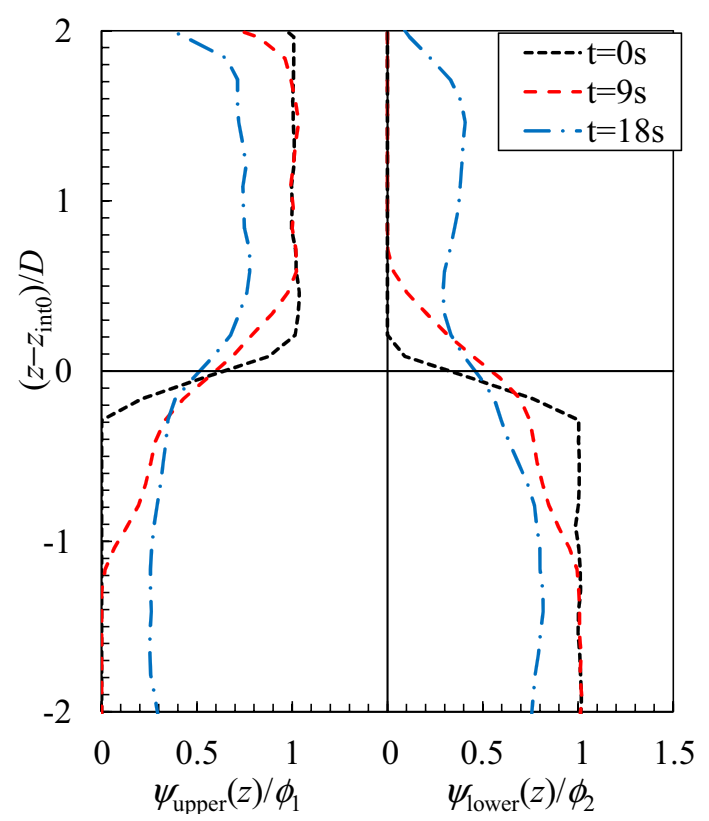

FIG. 7. Vertical distribution of particle volume density $\psi(z)$ for case No. $2 ; z_{\text {int0 }}$ is the initial interface height. 


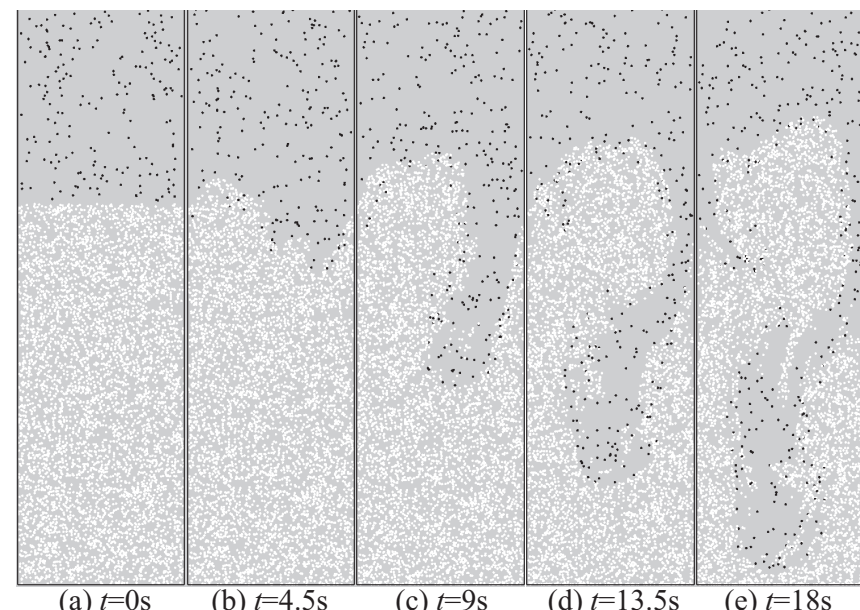

FIG. 8. Mixing behavior of low collective suspension of large particles on high collective suspension of small particles, case No. 3 (see the caption of Fig. 3).

the upper and lower suspensions can be seen by viewing the tip of the upper suspension's finger, and the particles in both suspensions cannot invade the other suspension individually because the collectivities of both upper and lower suspensions are high.

The vertical distribution of particle volume density in horizontal plane $\psi(z)$ is evaluated in this case by counting the number of particles in $T \times D \times 3 \Delta$ boxes for the upper and lower particles, respectively. Figure 7 shows the volume densities normalized by each suspension's volume fraction. The upper particle density just above the interface decreases over time, while that just below the interface increases, and vice versa for the lower particle density. At $t=18 \mathrm{~s}$, the density of the upper particles approaches that of the lower particles at the same height within $\pm 2 D$, confirming the macroscopic (vessel-scale) mixing.

Figure 8 shows the mixing behavior of a low collective $(C=5.70)$ suspension of large particles on a high collective $(C=14.36)$ suspension of small particles, in case No. 3 , and Fig. 9 is a magnified version of Fig. 8(d). We do not see the typical mushroom-type fingering settling in this case. Because the upper settling suspension's collectivity is low, the finger cannot have a clear interface. As shown in Fig. 9, the interface

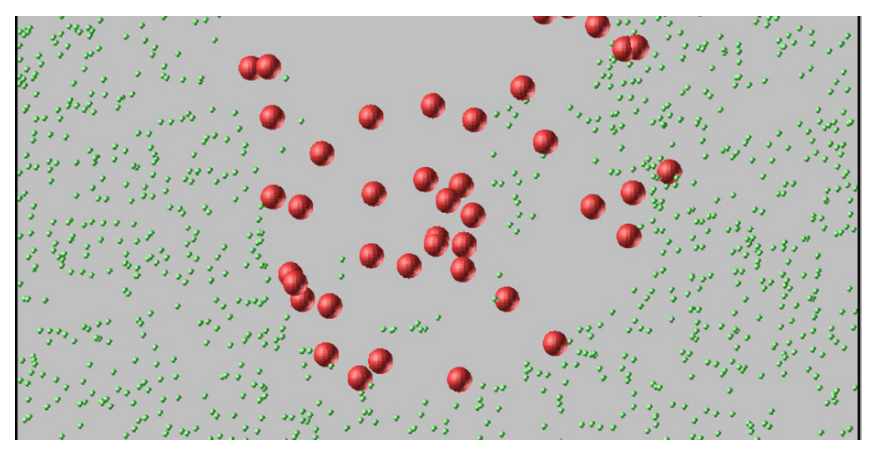

FIG. 9. Partial magnified image around the fingertip of Fig. 8(d) (see the caption of Fig. 4).

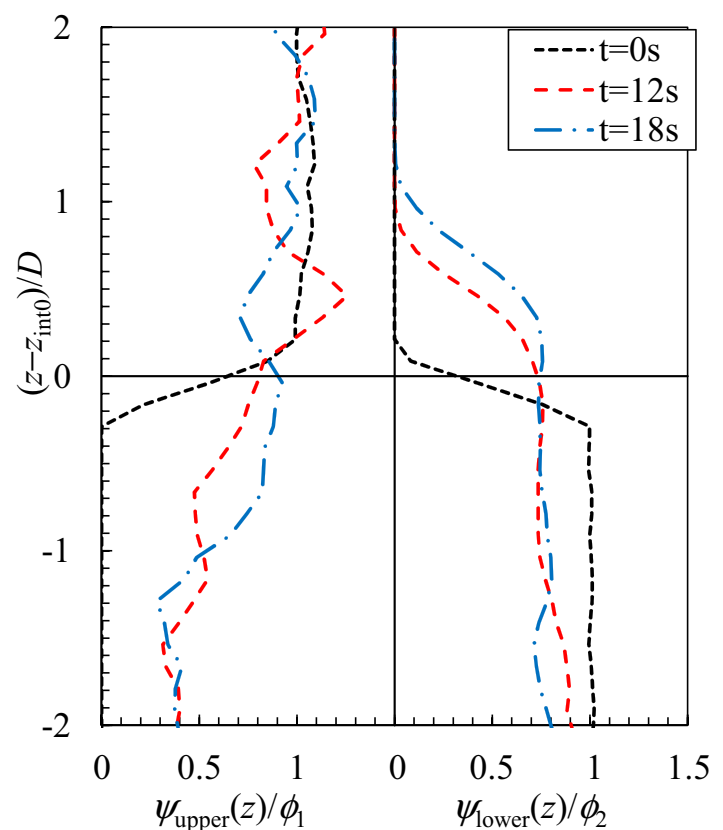

FIG. 10. Vertical distribution of particle volume density $\psi(z)$ for case No. 3.

cannot shield the microscopic particle mixing, so the upper particles invade the lower suspensions individually.

Figure 10 shows the vertical distribution of particle volume densities in this case. The upper particle infiltrates the lower suspension over time, but the lower suspension does not move upward so much. Because of the weak shielding of the interface, volume exchange slightly occurs.

Figure 11 shows the mixing behavior of a low collective $(C=3.33)$ suspension of large particles with relatively low apparent mass density on a high collective $(C=14.36)$ suspension of small particles with relatively high apparent mass density in case No. 4, and Fig. 12 depicts a partial magnification around the bottom-most upper particle of Fig. 11(e).

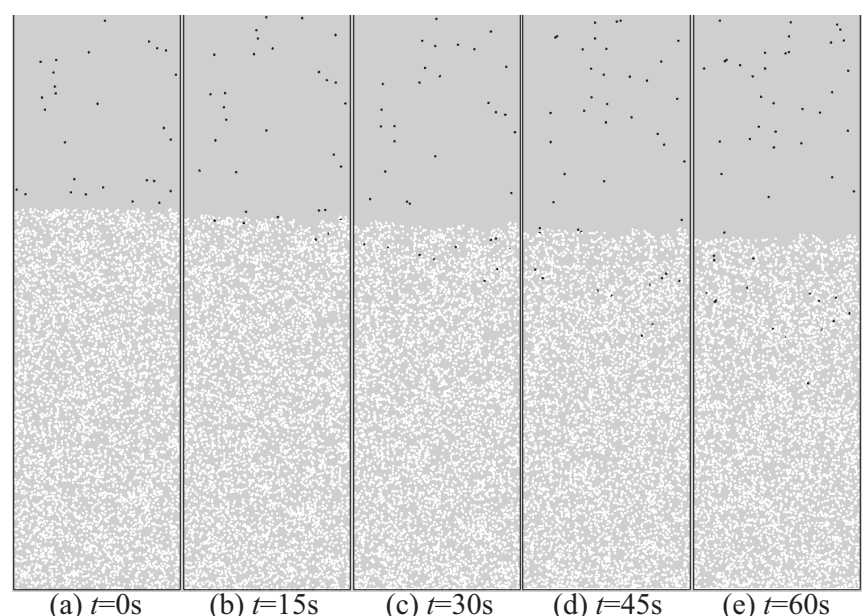

FIG. 11. Mixing behavior of low collective suspension of large particles with relatively low apparent mass density on high collective suspension of small particles with relatively high apparent mass density, case No. 4 (see the caption of Fig. 3). 


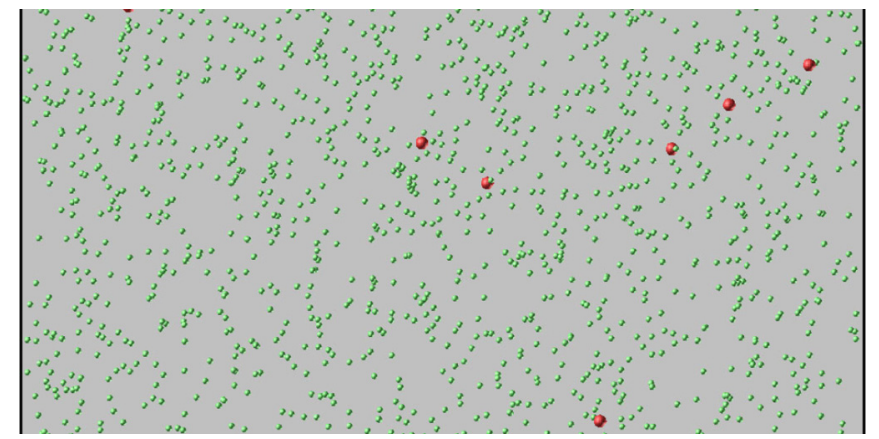

FIG. 12. Partial magnified image around the bottom-most upper particle of Fig. 11(e) (see the caption of Fig. 4).

Because this condition is gravitationally stable, we cannot see the fingering settling. Furthermore, because the terminal velocity of the upper particle is greater than that of the lower particle, the upper particles invade the lower suspension as individual particles rather than as a collective suspension, as illustrated in Fig. 12.

Figure 13 shows the vertical distribution of particle volume densities in this case. Because the mixing is only microscopic and caused by individual particles, it takes a very long time; and the upward flow of the lower suspension due to volume exchange does not occur.

Figure 14 shows the mixing behavior of a low collective $(C=8.22)$ suspension of large particles on a high collective $(C=14.36)$ suspension of small particles (case No. 5), and Fig. 15 depicts a partially magnified image around the fingertip of Fig. 14(e). The collectivity $C$ of the upper suspension is higher in this case than in case No. 3, but the interface between the upper and lower suspensions is more miscible, as shown in Figs. 8 and 14. Actually, the lower suspension conditions in both No. 3 and No. 5 are the same, as illustrated in Table I.

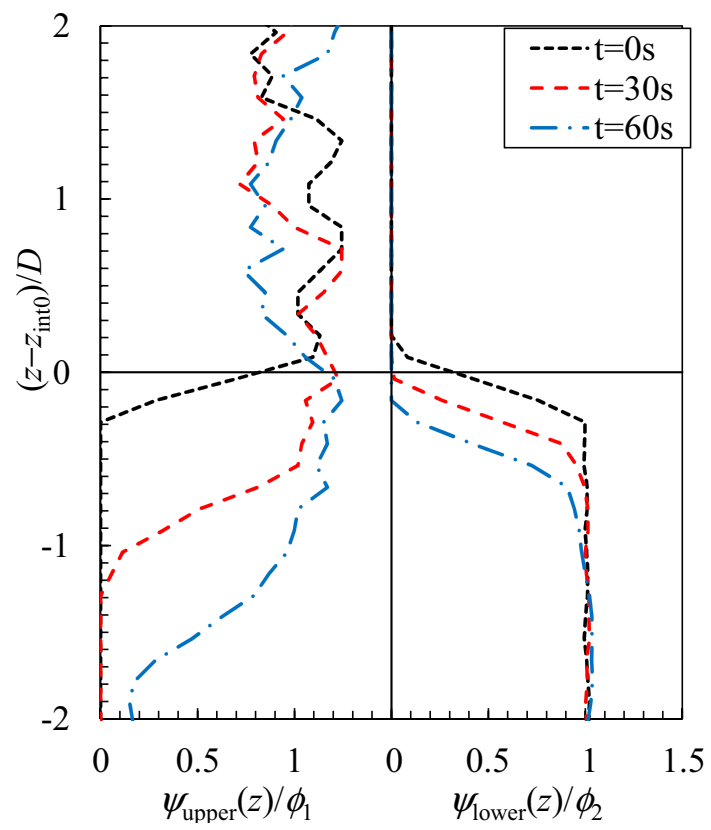

FIG. 13. Vertical distribution of particle volume density $\psi(z)$ for case No. 4 .

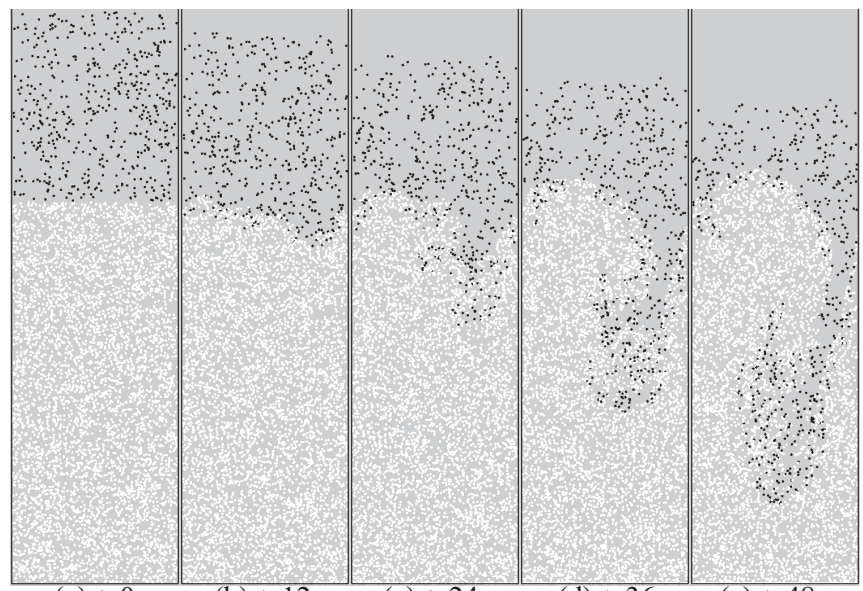

(a) $t=0 \mathrm{~s}$

(b) $t=12 \mathrm{~s}$

(c) $t=24 \mathrm{~s}$

(d) $t=36 \mathrm{~s}$

(e) $t=48 \mathrm{~s}$

FIG. 14. Mixing behavior of low collective suspension of large particles on high collective suspension of small particles, case No. 5 (see the caption of Fig. 3).

Comparing Figs. 9 and 15, we discovered that the interface in Fig. 15 cannot shield the particle microscopic mixing, so the mixing strength cannot be described solely by the magnitude of the collectivity. As a result, the suspensions' mixing behavior varies depending on the collectivity and terminal velocity. The miscibility of the suspensions' interface causes microscopic mixing and the immiscibility of the interface causes macroscopic mixing. In the following section, we will examine a unified description of these mixing behaviors at different spatial scales.

\section{UNIFIED MODEL OF PARTICLE MIXING}

For the five cases in Table I, Fig. 16 shows the time evolution of the mixing height $Z_{\text {mix }}$, which is defined as the vertical distance between the bottom-most particle of the upper suspension and the top-most particle of the lower suspension. With the exception of case No. 4, an individual particulate settling rather than collective fingering settling, $Z_{\text {mix }}$ increases exponentially at the start and then almost linearly thereafter. The broken lines represent the fitted linear lines and their slopes represent mixing speeds. The mixing speeds for those conditions vary depending on the mixing behavior, which can

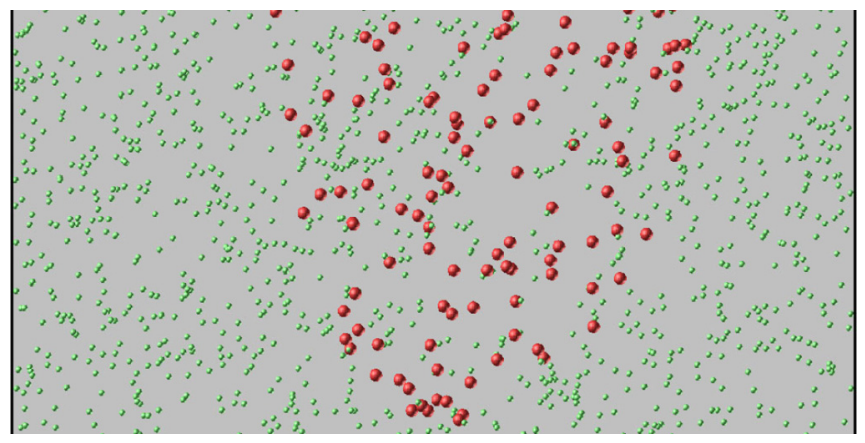

FIG. 15. Partial magnified image around the fingertip of Fig. 14(e) (see the caption of Fig. 4). 


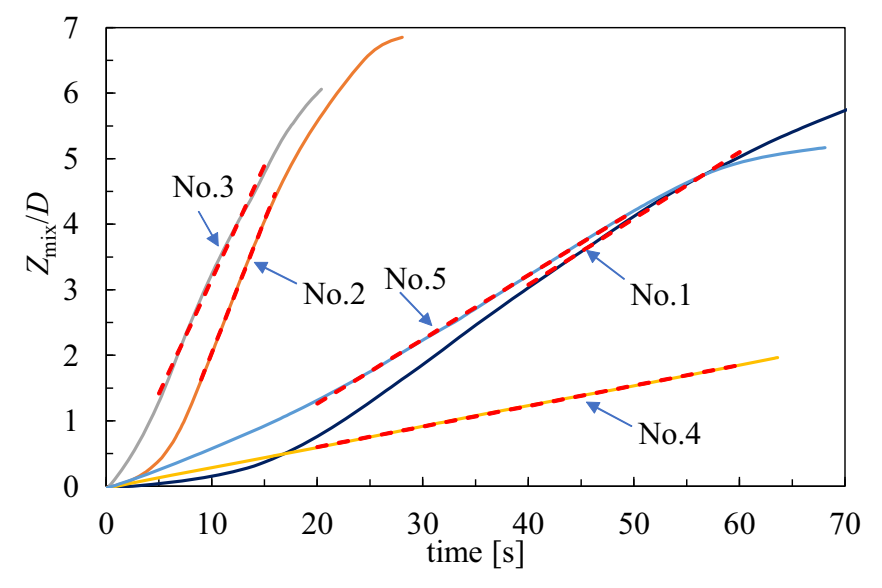

FIG. 16. Temporal development of mixing height $Z_{\text {mix }}$ for five cases (broken lines are linear fit of maximum slope region).

be macroscopic fingering mixing or microscopic individualparticle-scale mixing.

In this study, we attempted a theoretical approach that considers the collective suspension's settling velocity. When we assume that the upper suspension is nearly immiscible with the lower suspension, the settling velocity of a single fingerlike upper suspension blob in the lower suspension can be estimated in the same way as the Hadamard-Rybczynski solution of low Reynolds number fluid sphere settling [25] or Stokes terminal velocity, such as

$$
U_{\mathrm{f}} \sim \frac{\left(\rho_{\mathrm{s} 1}-\rho_{\mathrm{s} 2}\right)}{\mu_{l}} \lambda D g,
$$

where $U_{\mathrm{f}}$ is the settling velocity of the fingerlike upper suspension blob, and $\lambda$ is the finger width. $\rho_{\mathrm{s} 1}$ and $\rho_{\mathrm{s} 2}$ are the apparent mass density of the upper and lower suspensions, respectively, as

$$
\begin{aligned}
& \rho_{\mathrm{s} 1}=\phi_{1} \rho_{\mathrm{p} 1}+\left(1-\phi_{1}\right) \rho_{l}, \\
& \rho_{\mathrm{s} 2}=\phi_{2} \rho_{\mathrm{p} 2}+\left(1-\phi_{2}\right) \rho_{l} .
\end{aligned}
$$

where $\rho_{\mathrm{S}}$ is the apparent density of suspension, $\phi$ is the volume fraction of the particle, and subscripts 1 and 2 denote the upper and lower suspensions, respectively.

On the other hand, Sohn [20] derived the single-mode RT bubbling velocity of fluids (not suspensions) in the nonlinear regime in the cylindrical geometry as

$$
U_{\mathrm{RT}}=-k \frac{\mu_{\mathrm{up}}}{\rho_{\mathrm{up}}}+\sqrt{\frac{\left(\rho_{\mathrm{up}}-\rho_{\mathrm{low}}\right)}{\rho_{\mathrm{up}}} \frac{g}{k}+k^{2}\left(\frac{\mu_{\mathrm{up}}}{\rho_{\mathrm{up}}}\right)^{2}},
$$

where $k=2 \pi / L$ represents the single-mode dominant wave number ( $L$ is the cylinder diameter), and subscripts up and low are for upper and lower fluids. Further, we omitted the interfacial tension term. As discussed in [11-16], for unbounded turbulent RT mixing, the mixing speed is proportional to time $t$ and the penetration distance is proportional to $t^{2}$. However, for the single-mode RT mixing in the narrow channel, the penetration speed is constant, as shown by Eq. (9). Zanella et al. [21] applied a Taylor expansion to Eq. (9) for high viscosity conditions; then the bubble velocity can be expressed in our notation for zero interfacial tension as

$$
U_{\mathrm{RT}}=\frac{1}{8 \pi^{2}} \frac{\left(\rho_{\mathrm{up}}-\rho_{\mathrm{low}}\right)}{\mu_{\mathrm{up}}} L^{2} g .
$$

They performed the numerical simulation for a square channel, then confirmed that the theoretical velocity for the axisymmetric case could be applied for the square channel. It is interesting that Eq. (10) is a completely similar form to Eq. (6), although the derivation process is different.

Based on Eq. (6), we discuss the settling velocity of a fingerlike blob of the heavier suspension in the lighter suspension. According to Harada et al. [23], the finger width $\lambda$ in this aspect ratio vessel can be estimated to be of the order of $D$. The proportion constant of Eq. (6) is affected by the aspect ratio of the rectangular vessel, as discussed in the works of Harada et al. [23] and Kurose et al. [26]. We set the constant $K^{\prime}$ for the vessel in this study and substitute Eqs. (7) and (8) into Eq. (6),

$$
U_{\mathrm{f}}=K^{\prime} \frac{\phi_{1}\left(\rho_{\mathrm{p} 1}-\rho_{l}\right)-\phi_{2}\left(\rho_{\mathrm{p} 2}-\rho_{l}\right)}{\mu_{l}} D^{2} g .
$$

Here, from the right-hand side of Eq. (11), we can factor out the upper and lower particle terminal velocities as

$$
\begin{aligned}
U_{\mathrm{f}}=18 K^{\prime} & {\left[\left(\frac{D}{d_{\mathrm{p} 1}}\right)^{2} \phi_{1} \frac{\left(\rho_{\mathrm{p} 1}-\rho_{l}\right)}{18 \mu_{l}} d_{\mathrm{p} 1}^{2} g\right.} \\
- & \left.\left(\frac{D}{d_{\mathrm{p} 2}}\right)^{2} \phi_{2} \frac{\left(\rho_{\mathrm{p} 2}-\rho_{l}\right)}{18 \mu_{l}} d_{\mathrm{p} 2}^{2} g\right] .
\end{aligned}
$$

Then, the settling velocity of the fingerlike upper suspension blob nondimensionalized by the terminal velocity of the upper particle is obtained as

$$
\frac{U_{\mathrm{f}}}{U_{\mathrm{St} 1}}=K\left[\left(\frac{D}{d_{\mathrm{p} 1}} \phi_{1}^{1 / 2}\right)^{2}-\left(\frac{D}{d_{\mathrm{p} 2}} \phi_{2}^{1 / 2}\right)^{2} \frac{U_{\mathrm{St} 2}}{U_{\mathrm{St} 1}}\right],
$$

where $K=18 K^{\prime}$. The element $\left(D / d_{\mathrm{p}}\right) \phi^{1 / 2}$ is the modified collectivity of suspension as discussed in Mori et al. [22]. We set the nondimensional terms in square brackets in Eq. (13) to a parameter $Y_{\mathrm{m}}$ as

$$
Y_{\mathrm{m}}=\left(\frac{D}{d_{\mathrm{p} 1}} \phi_{1}^{1 / 2}\right)^{2}-\left(\frac{D}{d_{\mathrm{p} 2}} \phi_{2}^{1 / 2}\right)^{2} \frac{U_{\mathrm{St} 2}}{U_{\mathrm{St} 1}} .
$$

This nondimensional parameter includes both the upper and lower suspensions' collectivities as well as the upper and lower particle terminal velocities, so it affects both macroscopic and microscopic mixing speeds. Then, we will consider the mixing speed by using this nondimensional parameter $Y_{\mathrm{m}}$.

We calculated the mixing speed $d Z_{\text {mix }} / d t$ from the slope of $Z_{\text {mix }}$ in a linearly increasing region, for five cases in Fig. 16 and for the other 34 cases (see Supplemental Material [27]), and plotted $d Z_{\text {mix }} / d t$ nondimensionalized by the upper particle terminal velocity $U_{\mathrm{St} 1}$ versus $Y_{\mathrm{m}}$ in Fig. 17 . Note that all the data shown in Fig. 17 were obtained by numerical simulations, although Mori et al. [22] conducted experimental observations partially corresponding to the present study. Because the tip position of the upward finger (spike) of the lower suspension cannot be observed from outside of the vessel in the experiment, we discuss the mixing rate by using numerical results. 


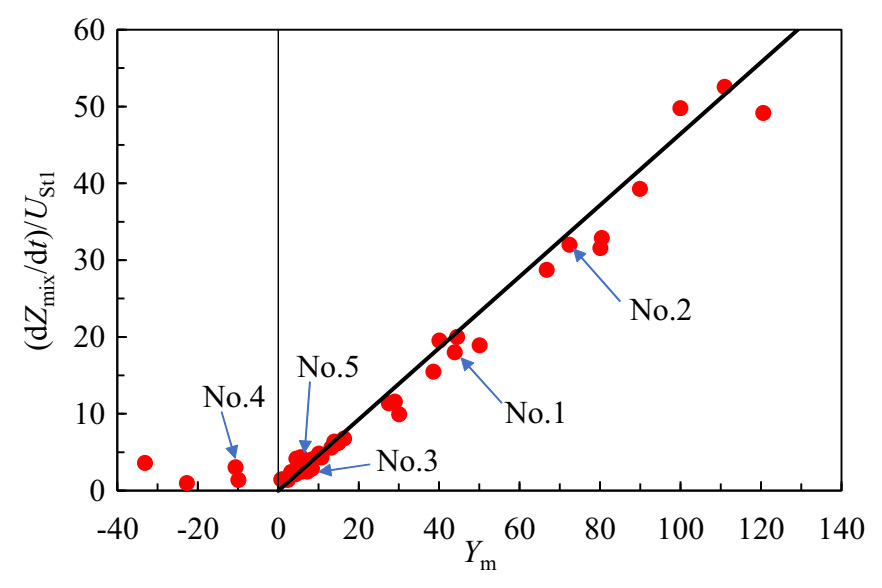

FIG. 17. Relation between mixing speed $d Z_{\text {mix }} / d t$ and nondimensional number $Y_{\mathrm{m}}$ for 39 cases (bold solid line represents the estimated relation using experimentally fitted finger settling speed).

The conditions causing only microscopic particulate mixing in this figure are in the negative region of $Y_{\mathrm{m}}$. As shown in Eq. (6), the sign of $Y_{\mathrm{m}}$ corresponds to the sign of $\left(\rho_{\mathrm{s} 1}-\rho_{\mathrm{s} 2}\right)$, with $Y_{\mathrm{m}} \leqslant 0$ corresponding to a gravitationally stable condition and $Y_{\mathrm{m}}>0$ corresponding to a gravitationally unstable (Rayleigh-Taylor) condition.

The conditions with macroscopic mixing by collective settling, on the other hand, are in the positive $Y_{\mathrm{m}}$ region, and the nondimensional mixing speed is proportional to $Y_{\mathrm{m}}$. The microscopic mixing speed is small and comparable to the single-particle terminal velocity. The macroscopic mixing speed can be estimated by the fingering settling speed and the almost similar speed of the upflow due to volume exchange, which we estimate as twice the finger settling speed and from Eqs. (13) and (14),

$$
\frac{d Z_{\mathrm{mix}}}{d t} \frac{1}{U_{\mathrm{St} 1}} \sim 2 \frac{U_{\mathrm{f}}}{U_{\mathrm{St} 1}}=2 K Y_{\mathrm{m}}
$$

Zanella et al. [21] show the vertical symmetric behavior in the high viscosity case RT mixing in a confined vessel, so the mixing speed can be estimated as twice the bubble velocity. In Fig. 17, the relation of Eq. (15) is shown by the bold solid line. Mori et al. [22] obtained the coefficient $K$ of that line by fitting the experimental data within $30 \leqslant\left(d_{\mathrm{p}} / D\right)^{-2}\left(\phi_{1}-\phi_{2}\right)<$ 120 , which is in the same vessel condition as in this study and $\left(d_{\mathrm{p}} / D\right)^{-2}\left(\phi_{1}-\phi_{2}\right)$ corresponds to $Y_{\mathrm{m}}$ with homogeneous particles $(2 K=0.465)$. In our study, the mixing speed data obtained by the numerical experiments with positive $Y_{\mathrm{m}}$ almost agree with the relation by Eq. (15), indicating that the parameter $Y_{\mathrm{m}}$ can determine the speed of the macroscopic mixing. This stratified suspensions mixing is determined by the properties of the suspensions and particles, rather than by the concentration gradient as in Fickian mixing. The coefficient $K$ in Eq. (15) can be estimated using the Harada et al. [23] experimental relationship for the other vessel conditions. In addition, as a reference in different configurations assuming the axisymmetric case, we compute the coefficient $K$ by using Eq. (10) with the narrower depth $L=D$ and obtained $2 K=2\left(18 / 8 \pi^{2}\right)=0.456$, which is very close to our results. Thus, using only one parameter $Y_{\mathrm{m}}$, we can predict the mix- ing behavior and estimate the mixing speed of the stratified suspensions.

\section{SUMMARY}

We used a point-force two-way coupling method to conduct numerical experiments to investigate the mixing of stratified suspensions containing different types of particles. We studied the mixing behavior of stratified suspension in the vessel's center plane and discovered two types of mixing: microscopic (individual-particle-level) mixing and macroscopic (vessel-scale) collective mixing, which differed from Fickian mixing. In addition, we investigated the vertical mixing speed of the stratified suspension. We used a simple theoretical model to analyze the fingering settling velocity. Then we introduced a nondimensional number $Y_{\mathrm{m}}$, which represents the difference in collectivities of the upper and lower suspensions while accounting for both upper and lower particle terminal velocities. We discovered that the negative sign of the proposed nondimensional parameter $Y_{\mathrm{m}}$ could distinguish only microscopic mixing, whereas the positive value of $Y_{\mathrm{m}}$ could predict macroscopic mixing speed.

\section{ACKNOWLEDGMENTS}

The authors gratefully acknowledge R. Otomo, K. Nishimura, and G. Masuda for insightful discussions. This study was supported by Japan Society for the Promotion of Science KAKENHI Grant No. 19H02058.

\section{APPENDIX: RESOLUTION DEPENDENCY TEST}

We compared the results obtained by three resolution conditions, i.e., low $(4 \times 8 \times 32)$, middle $(8 \times 16 \times 64)$, and high $(16 \times 32 \times 128)$, for $D \times T \times L$. The time steps for each cases were adjusted to 10,5 , and $2.5 \times 10^{-5} \mathrm{~s}$ for the low,

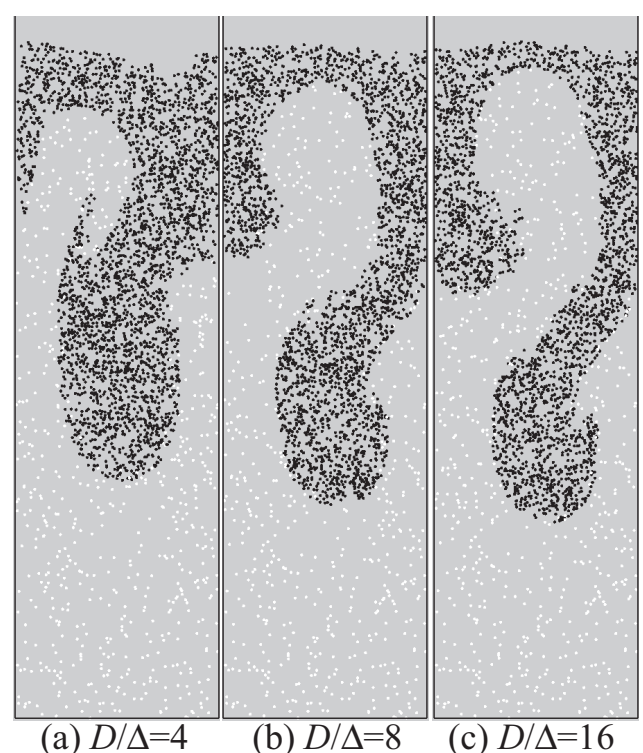

FIG. 18. Comparison of grid resolutions for snapshots of case No. 1 at $t=50 \mathrm{~s}$. $D$ is the depth of the vessel and $\Delta$ is grid spacing (see the caption of Fig. 3). 
middle, and high resolution, respectively. Figure 18 shows the instantaneous particle distribution at $t=50 \mathrm{~s}$ in the center plane region with depth of $0.1 D$, which is the same as Fig. 3. The finger shape obtained by the low resolution case Fig. 3(a) is different from the other results [Figs. 3(b) and 3(c)]; however, the middle case result [Fig. 3(b)] is almost the same as that by the high resolution [Fig. 3(c)]. A nondi- mensional mixing speed $\left(d Z_{\operatorname{mix}} / d t\right) / U_{\mathrm{St} 1}$ is also compared for those three conditions and obtained as 17.3, 18.0, and 18.4 for the low, middle, and high resolution, respectively. The low resolution result is about $6 \%$ different from the high resolution case; however, the middle resolution result is only $2 \%$ less than the high resolution case. Thus we considered the middle resolution condition to be satisfactorily usable in this study.
[1] R. H. Davis, and A. Acrivos, Sedimentation of noncolloidal particles at low Reynolds numbers, Annu. Rev. Fluid Mech. 17, 91 (1985).

[2] G. J. Kynch, A theory of sedimentation, Trans. Faraday Soc. 48, 166 (1952).

[3] A. E. Boycott, Sedimentation of blood corpuscles, Nature (London) 104, 532 (1920).

[4] C. Völtz, W. Pesch, and I. Rehberg, Rayleigh-Taylor instability in a sedimenting suspension, Phys. Rev. E 65, 011404 (2001).

[5] S. Harada, T. Mitsui, and K. Sato, Particle-like and fluidlike settling of a stratified suspension, Eur. Phys. J. E 35, 1 (2012).

[6] G. Machu, W. Meile, L. C. Nitsche, and U. Schaflinger, Coalescence, torus formation and breakup of sedimenting drops: Experiments and computer simulations, J. Fluid Mech. 447, 299 (2001).

[7] T. Bosse, L. Kleiser, C. Härtel, and E. Meiburg, Numerical simulation of finite Reynolds number suspension drops settling under gravity, Phys. Fluids 17, 037101 (2005).

[8] B. Metzger, M. Nicolas, and É. Guazzelli, Falling clouds of particles in viscous fluids, J. Fluid Mech. 580, 283 (2007).

[9] J. M. Nitsche and G. K. Batchelor, Breack-up of a falling drop containing dispersed particles, J. Fluid Mech. 340, 161 (1997).

[10] R. M. Davies and G. I. Taylor, The mechanics of large bubbles rising through liquids in tubes, Proc. R. Soc. London A 200, 375 (1950).

[11] D. L. Youngs, Numerical simulation of turbulent mixing by Rayleigh-Taylor instability, Phys. D 12, 32 (1984).

[12] D. H. Sharp, An overview of Rayleigh-Taylor instability, Phys. D 12, 3 (1984).

[13] G. Dimonte and M. Schneider, Density ratio dependence of Rayleigh-Taylor mixing for sustained and impulsive acceleration histories, Phys. Fluids 12, 304 (2000).

[14] G. Dimonte, Spanwise homogeneous buoyancy-drag model for Rayleigh-Taylor mixing and experimental evaluation, Phys. Plasmas 7, 2255 (2000).
[15] P. Ramaprabhu and M. J. Andrews, Experimental investigation of Rayleigh-Taylor mixing at small Atwood numbers, J. Fluid Mech. 502, 233 (2004).

[16] J. Glimm, D. H. Sharp, T. Kaman, and H. Lim, New directions for Rayleigh-Taylor mixing, Philos. Trans. R. Soc. A 371, 20120183 (2013).

[17] D. Layzer, On the instability of superposed fluids in a gravitational field, Astrophys. J. 122, 1 (1955).

[18] A. G. W. Lawrie, Rayleigh-Taylor mixing: Confinement by stratification and geometry, Ph.D. thesis, The University of Cambridge, 2009.

[19] J. P. Wilkinson, and J. W. Jacobs, Experimental study of the single-mode three-dimensional Rayleigh-Taylor instability, Phys. Fluids 19, 124102 (2007).

[20] S.-I. Sohn, Effects of surface tension and viscosity on the growth rates of Rayleigh-Taylor and Richtmyer-Meshkov instabilities, Phys. Rev. E 80, 055302(R) (2009).

[21] R. Zanella, G. Tegze, R. Le Tellier, and H. Henry, Twoand three-dimensional simulations of Rayleigh-Taylor instabilities using a coupled Cahn-Hilliard/Navier-Stokes model, Phys. Fluids 32, 124115 (2020).

[22] M. Mori, T. Tai, K. Nishimura, S. Harada, and Y. Yamamoto, Possibility of non-Fickian mixing at concentration interface between stratified suspensions, J. Colloid Interface Sci. 571, 13 (2020).

[23] S. Harada, M. Kondo, K. Watanabe, T. Shiotani, and K. Sato, Collective settling of fine particles in a narrow channel with arbitrary cross-section, Chem. Eng. Sci. 93, 307 (2013).

[24] Y. Yamamoto, F. Hisataka, and S. Harada, Numerical simulation of concentration interface in stratified suspension: Continuumparticle transition, Intl. J. Multiphase Flow 73, 71 (2015).

[25] R. C. Clift, B. J. Grace, and M. E. Weber, Bubbles, Drops, and Particles (Academic Press, New York, 1978).

[26] Y. Kurose, K. Ishizawa, M. R. Soriano, and S. Harada, Scaleindependent model of gravitational dispersion of particulate suspension in fractal channel, Chem. Eng. Sci. 199, 231 (2019).

[27] See Supplemental Material at http://link.aps.org/supplemental/ 10.1103/PhysRevE.104.025111 for all condition data. 